\title{
Reversible hydrogenation restores defected graphene to graphene
}

\author{
Lin Jiang ${ }^{1}$, Pauline M.G. van Deursen ${ }^{1}$, Hadi Arjmandi-Tash ${ }^{1}$, Liubov A. Belyaeva ${ }^{1}$, \\ Haoyuan $\mathrm{Qi}^{2,5}$, Jiao $\mathrm{He}^{3}$, Vincent Kofman ${ }^{3}$, Longfei $\mathrm{Wu}^{4}$, Valery Muravev ${ }^{4}$, Ute Kaiser ${ }^{2}$, \\ Harold Linnartz ${ }^{3}$, Emiel J. M. Hensen ${ }^{4}$, Jan P. Hofmann ${ }^{4}$ \& Grégory F. Schneider ${ }^{1 *}$ \\ ${ }^{1}$ Leiden Institute of Chemistry, Leiden University, Einsteinweg 55, 2333CC Leiden, The Netherlands; \\ ${ }^{2}$ Central Facility for Electron Microscopy, Electron Microscopy Group of Materials Science, Ulm University, \\ Albert-Einstein-Allee 11, Ulm 89081, Germany; \\ ${ }^{3}$ Laboratory for Astrophysics, Leiden Observatory, Leiden University, Niels Bohrweg 2, 2333 CA Leiden, The Netherlands; \\ ${ }^{4}$ Laboratory for Inorganic Materials and Catalysis, Department of Chemical Engineering and Chemistry, Eindhoven University of Technology, \\ P.O. Box 513, 5600 MB Eindhoven, The Netherlands; \\ ${ }^{5}$ Center for Advancing Electronics Dresden (cfaed) and Faculty of Chemistry and Food Chemistry, Technical University of Dresden, \\ 010069 Dresden, Germany
}

Received October 17, 2020; accepted February 5, 2021; published online April 25, 2021

\begin{abstract}
Graphene as a two-dimensional material is prone to hydrocarbon contaminations, which can significantly alter its intrinsic electrical properties. Herein, we implement a facile hydrogenation-dehydrogenation strategy to remove hydrocarbon contaminations and preserve the excellent transport properties of monolayer graphene. Using electron microscopy we quantitatively characterized the improved cleanness of hydrogenated graphene compared to untreated samples. In situ spectroscopic investigations revealed that the hydrogenation treatment promoted the adsorption of water at the graphene surface, resulting in a protective layer against the re-deposition of hydrocarbon molecules. Additionally, the further dehydrogenation of hydrogenated graphene rendered a more pristine-like basal plane with improved carrier mobility compared to untreated pristine graphene. Our findings provide a practical post-growth cleaning protocol for graphene with maintained surface cleanness and lattice integrity to systematically carry a range of surface chemistry in the form of a well-performing and reproducible transistor.
\end{abstract}

graphene, reversible hydrogenation, surface cleanness, in situ spectroscopy, transistors

Citation: Jiang L, Deursen PMG, Arjmandi-tash H, Belyaeva LA, Qi H, He J, Kofman V, Wu L, Muravev V, Kaiser U, Linnartz H, Hensen EJM, Hofmann JP, Schneider GF. Reversible hydrogenation restores defected graphene to graphene. Sci China Chem, 2021, 64: 1047-1056, https://doi.org/10.1007/ s11426-020-9959-5

\section{Introduction}

Graphene applications require a high cleanness and a precise knowledge of the chemical structure of the basal plane [1-4]. Surface contaminations are generally introduced during chemical vapor deposition (CVD) growth $[5,6]$, post-growth transfer [7] and air exposure [8]. However, they can alter the intrinsic properties and performances of CVD graphene, e.g.,

*Corresponding author (email: g.f.schneider@chem.leidenuniv.nl) large resistance fluctuations and batch-to-batch variations [7-10]. In fact, amorphous hydrocarbon, which can originate from both the CVD growth process [5] and any ambient environment, is one of the most encountered contaminants. Such hydrophobic contaminants can drastically alter the wettability [8] and the electrochemistry [11,12] of graphene. To date, quite a few post-growth methods have been explored to eliminate these surface contaminants, such as current annealing [13], mechanical removal [14,15], chemical etching [16], and plasma cleaning [17,18]. Among 
them hydrogen plasma technique has the advantage of largescale applicability and uniformity $[19,20]$. Most importantly, the introduced hydrogenated defects $\left(\mathrm{H}-s p^{3}\right.$ sites) can be completely removed by annealing, thus leading to a recovered high carrier mobility of the dehydrogenated graphene [21].

Here, we employed a mild hydrogen plasma on graphene followed by dehydrogenation and restoring the $s p^{3}$ defect sites to $s p^{2}$ for yielding cleaner graphene samples. The mild hydrogen plasma, which was initially designated to chemically functionalize graphene (referred to as $\mathrm{H}-\mathrm{G}$ ) in previous work $[12,22]$, was employed to clean off the surface hydrocarbons. Statistical analysis of aberration-corrected highresolution transmission electron microscopic images (ACHRTEM) confirmed that hydrogenated graphene maintained a good lattice integrity and enhanced surface cleanness (less hydrocarbon contaminations) compared to untreated, but hydrocarbons-contaminated, graphene. As a result, we hypothesized that, in addition to chemically modifying the basal plane from $s p^{2}$ to $s p^{3}$, hydrogenation also cleans graphene by removing surface adsorbed hydrocarbons. Furthermore, in situ spectroscopic techniques including temperature programmed desorption-infrared spectroscopy (TPD-IR) and near-ambient pressure X-ray photoelectron spectroscopy (NAP-XPS) demonstrated a layer of water adsorbed on the surface of hydrogenated graphene preserving the cleanness of the basal planes, as predicted $[12,23]$. Additionally, the dehydrogenation of hydrogenated graphene yields, reversibly, the original $s p^{2}$ hybridized lattices with improved surface cleanness and high carrier mobility compared to untreated graphene.

\section{Experimental}

\subsection{Materials and chemicals}

CVD graphene was purchased from Graphenea SA. Poly (methyl methacrylate) (Sigma Aldrich) was used to transfer CVD graphene. Potassium chloride $(\mathrm{KCl}, \geq 99.0 \%$ ), ammonium persulfate $\left(\left(\mathrm{NH}_{4}\right)_{2} \mathrm{~S}_{2} \mathrm{O}_{8}, \geq 98.0 \%\right)$ and tris-buffer (2amino-2-hydroxymethyl-propane-1,3-diol) were purchased from Sigma Aldrich. The electrolyte solution was prepared with ultrapure water (Millipore Milli-Q gradient A10 system, $18.2 \mathrm{M} \Omega \mathrm{cm}$ ).

\subsection{Plasma conditions}

A capacitively coupled plasma system with a radio-frequency (RF) of $40 \mathrm{kHz}$ and a base pressure below $0.02 \mathrm{mbar}$ (Diener electronic, Femto) was employed at room temperature. The power/pressure ratio used for controlled surface modifications was $10 \mathrm{~W} / 1.0$ mbar for hydrogen plasma and $8 \mathrm{~W} / 0.85$ mbar for argon plasma, respectively. Inside the plasma chamber, a perforated Faraday cage was used to shield energetic hydrogen ions to form mild radical plasma to react with graphene. In detail, argon plasma composed of ions can physically bombard the carbon lattices [24] while hydrogen plasma (in combination with the Faraday cage) containing chemically reactive radicals was expected to react with and functionalize the lattices [19] (Scheme 1).

\section{3 (HR)TEM characterization}

CVD graphene (Graphenea) was employed to prepare the samples. We deliberately opted for commercial graphene to assure reproducibility. No extra polymer was used to transfer graphene onto TEM grids. After rinsing the grid and CVD graphene on copper foil with acetone and isopropanol, a droplet of ultrapure water (Millipore Milli-Q gradient A10 system, 18.2 $\mathrm{M} \Omega \mathrm{cm}$ ) was added between the grid and graphene-copper foil. After the water dried out graphene was then attached to the grid, following the chemical etching of copper foil in $0.5 \mathrm{M}$ ammonium persulfate solution. ACHRTEM imaging was conducted on an FEI Titan 80-300 microscope at an acceleration voltage of $80 \mathrm{kV}$ (Ulm University). TEM images were collected on a Tecnai F20 microscope (Thermo-Fisher Scientific) operating at $200 \mathrm{kV}$ (Leiden University Medical Center, LUMC).

\subsection{In situ TPD-FTIR}

The temperature programmed desorption-infrared spectroscopy (TPD-IR) was performed in a custom-made high vacuum system, comprising a central high vacuum chamber with a closed-cycle helium refrigerator mounted on top. Water deposition on $\mathrm{abF}_{2}$ window mounted on the tip of a cold finger was monitored using a Fourier transform infrared (FTIR) spectrometer. IR spectra in the range of $700-4,000 \mathrm{~cm}^{-1}$ and with a resolution of $1 \mathrm{~cm}^{-1}$ were collected in transmission mode at normal incidence every $90 \mathrm{~s}$. CVD graphene films were transferred onto the $\mathrm{BaF}_{2}$ window via a biphasic method developed by the co-authors [25].

\subsection{In situ NAP-XPS}

NAP-XPS spectra were recorded with a SPECS XPS spec-

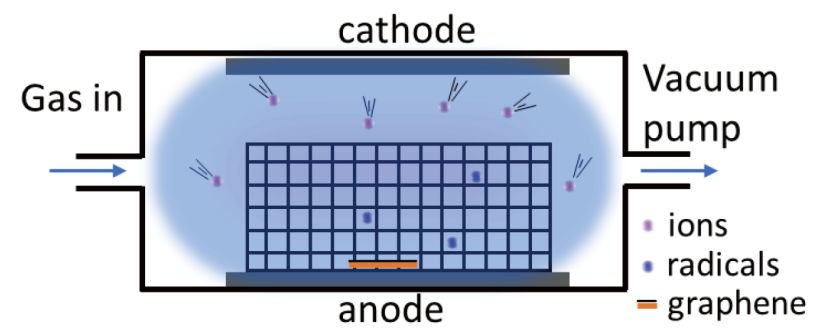

Scheme 1 Illustration of a hydrogen plasma reactor with a perforated Faraday cage (color online). 
trometer equipped with a monochromatic small-spot $(300 \mu \mathrm{m}) \mathrm{X}$-ray source, an $\mathrm{Al}$ anode $\left(\mathrm{Al} K_{\alpha}=1,486.6 \mathrm{eV}\right)$ and a $180^{\circ}$ double-focusing hemispherical analyzer working with a multichannel 1D delay-line detector. XPS measurements at pressures of up to 20 mbar were possible using a differential pumping system, which separates the electron analyzer (SPECS Phoibos NAP-150) from the reaction area via a nozzle with an aperture of $300 \mu \mathrm{m}$. Filling of the NAPcell with water vapor was performed by means of piezoelectric leak valve. The water reservoir was kept at room temperature. Further details about the design of the NAPXPS cell can be found in literature [26]. Ultra-high vacuum (UHV) measurements were conducted at a chamber pressure of $<10^{-9}$ mbar. XP spectra were processed by CasaXPS software using Shirley background subtraction.

\subsection{Raman characterizations}

Raman spectra were collected on a WITEC alpha300 R confocal Raman imaging with a laser wavelength of $532 \mathrm{~nm}$. Laser power was set to $<2 \mathrm{~mW}$ to minimize potential damages from laser induced heating of the samples. All measurements were performed under ambient conditions at room temperature.

\subsection{GFET fabrication and electrical measurements}

CVD graphene grown on copper foil was first hydrogenated for $60 \mathrm{~s}$ and then annealed at $350{ }^{\circ} \mathrm{C}$ (under argon atmo- sphere) for $1 \mathrm{~h}$ to dehydrogenate the H-G. A traditional polymer (polymethyl methacrylate, PMMA) assisted method was employed to transfer graphene onto a silicon wafer with a 300-nm thick oxide layer on top. Afterwards, the gold/ chromium $(50 \mathrm{~nm} / 5 \mathrm{~nm})$ electrodes were thermally deposited on graphene- $\mathrm{SiO}_{2} / \mathrm{Si}$, functioning as the source (S) and drain (D) electrodes. The dimensions of graphene channel were about $2-3 \mathrm{~mm}$ in length and $1.4-5 \mathrm{~mm}$ in width. Clean epoxy resin was used to seal the electrodes. A SR830 DSP lock-in amplifier was employed to measure the transport characteristics of graphene field effect transistors (GFET) devices. Electrolyte-gating voltage was applied by a reference electrode (Radiometer Analytical Red Rod) through a solution of $0.1 \mathrm{M} \mathrm{KCl}$ containing $10 \mathrm{mM}$ Tris as the buffer ( $\mathrm{pH}$ 8). A fixed alternating current (AC) of $1 \mu \mathrm{A}$ was applied between the source and drain electrode.

\section{Results and discussion}

\subsection{Cleaning effect of hydrogenated graphene}

The cleaning effect of hydrogen plasma on graphene films grown by a CVD method [27] was studied by AC-HRTEM. Hydrogenated graphene (H-G) was obtained from untreated graphene upon $60 \mathrm{~s}$ of hydrogen plasma treatment. The asreceived graphene without plasma treatment (referred to asuntreated G) was used as a control sample. Figure 1(a) shows the AC-HRTEM images of untreated $\mathrm{G}$ with the lattice fully covered with amorphous contaminations which are mainly
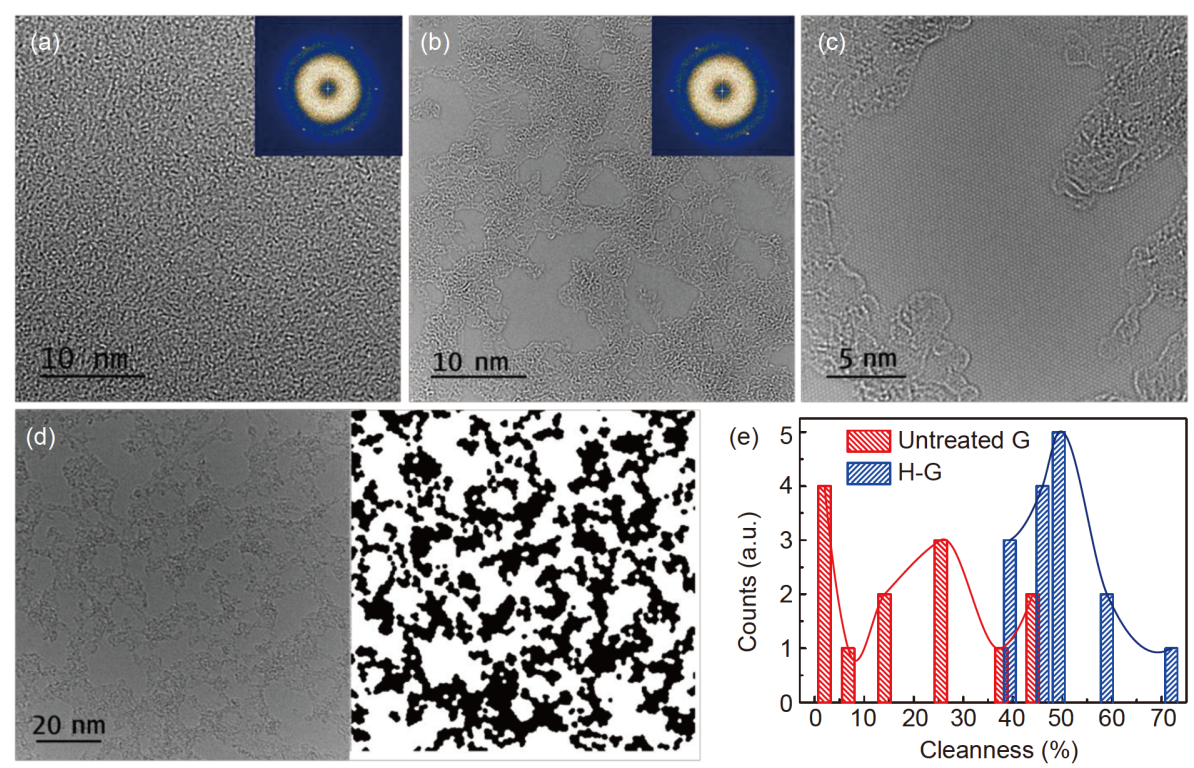

Figure 1 Improved surface cleanness of graphene upon hydrogenation. (a) HRTEM image of untreated graphene. The inset of FFT patterns shows six reflections at $4.7 \mathrm{~nm}^{-1}$, demonstrating the crystallinity of monolayer graphene. (b) HRTEM image of hydrogenated graphene (H-G) after $60 \mathrm{~s}$ of hydrogenation of untreated graphene $(\mathrm{G})$, showing a decreased level of contaminations. The six reflections of FFT patterns (scale bar: 4.7 nm ${ }^{-1}$ ) indicate the preservation of graphene lattice integrity. (c) A zoomed-in HRTEM image of H-G. (d) A TEM image of H-G (60 s) and its corresponding binary image distinguishing the clean and contaminated areas, respectively, with white and black colors. The cleanness ( $59 \%)$ is derived from dividing the number of white pixels by the total number of pixels in the image. (e) Cleanness comparison for untreated G vs. H-G (color online). 
composed of hydrocarbons [28,29]. The first-order reflection in the fast Fourier transform (FFT) patterns (in the inset) indicates the preserved crystallinity of monolayer graphene. By contrast, H-G in Figure 1(b) shows larger visible areas of the graphene lattice surrounded by less amorphous patterns. The preserved crystallinity of H-G is confirmed by the six reflections FFT patterns in the inset. Particularly, a magnified HRTEM of H-G in Figure 1(c) confirms the clean and wellpreserved lattice integrity in $\mathrm{H}-\mathrm{G}$.

To further evaluate the cleaning effect by hydrogenation, we prepared a graphene sample treated with argon plasma (Ar-G) containing a comparable level of defect density as H$\mathrm{G}$ (Figure S1) [12]. As shown in Figure S2, the cleanness of $\mathrm{H}-\mathrm{G}$ is estimated to be as $\sim 3$-fold as that for Ar-G at similar magnifications. More importantly, the surface of H-G exhibits a clear contrast between the clean lattice and amorphous patterns, which allows for further statistical analysis of lattice cleanness. Such cleaning contrasts between hydrogen and argon plasma should mainly be related to the plasma conditions employed in this work. In fact, the energies of plasma particles (i.e. ions, radicals) determine the degree of functionalization, defect generation or etching of graphene lattices, and/or cleaning effect by removing hydrocarbons adsorbed on the surface of graphene [20,30]. Argon plasma, mainly composed of energetic argon ions, physically bombards the carbon lattice to generate vacancy defects, reflected by the $\mathrm{D}$ peak in Raman spectra and the reported $I(\mathrm{D}) / I\left(\mathrm{D}^{\prime}\right)$ ratio of seven (Figure S1) [31]. By contrast, hydrogen plasma (with the graphene sample in a Faraday cage) mainly introduces $s p^{3}$ defects (Figure S1) and cleans off hydrocarbons from the lattice [12]. From the overall cleanness comparison, we conclude that, without sacrificing the conjugated carbon network and crystallinity, the hydrogen plasma cleans off the amorphous contaminants on the graphene surface and uniformly hybridizes graphene lattice from $s p^{2}$ to $s p^{3}$.

All the TEM characterizations were performed within three to five days after the sample preparation using a nonpolymer transfer method [2,32]. In view of the cleanness contrast between $\mathrm{H}-\mathrm{G}$ and $\mathrm{G}$ in Figure 1, it is concluded that the cleaned $\mathrm{H}-\mathrm{G}$ is, to a certain degree, resistant to the recontamination of hydrocarbons. When the ambient exposure time extends to longer than two weeks, no contrast in surface morphology between $\mathrm{H}-\mathrm{G}$ and untreated $\mathrm{G}$ was observed (Figure S3). The mechanism responsible for such a behavior was therefore investigated further.

To gain a quantitative insight into the cleaning effect upon hydrogenation, a statistical analysis of the TEM images of graphene acquired with a larger field-of-view was performed. As contaminations appear in patterns with more rough sites while smooth clean lattice in $\mathrm{H}-\mathrm{G}$, the cleanness within the field-of-view, could therefore quantitatively be evaluated by converting the TEM image into a black-white binary image. We first developed a MATLAB script (see
Supplementary Note 1) to process TEM micrographs and to identify the surface contaminations automatically. Table S1 details the process within the script. Figure 1(d) illustrates a selected region in the TEM image of H-G before (left) and after (right) image processing. The black pixels in the right panel correspond to the identified contaminations in graphene. Using the processed images, the graphene "cleanness" is defined as the number of pixels representing the clean area divided by the total number of pixels in the image. Specifically, the cleanness of the image is calculated as the sum of the number of white pixels (clean graphene area) normalized by the total number of pixels. Based on a statistical analysis of thirteen to fifteen images for both $G$ and $\mathrm{H}-\mathrm{G}$, the quantitative evaluation of the cleanness summarized in Figure 1(e) indicates that contaminations on the surface of H-G (average cleanness: 50\%) are considerably less than that of untreated $\mathrm{G}$ (average cleanness: $20 \%$ ). In particular, the cleanness of $\mathrm{H}-\mathrm{G}$ ranging from $40 \%-72 \%$ is superior to previous work using dry-cleaning method with a cleanness of $15 \%-40 \%$ [28]. Moreover, the amorphous contaminations exhibit a thinner and more homogeneous morphology after hydrogenation than that of untreated $G$ (Figure 1(a)) and Ar-G (Figure S1). This is the first time to quantitatively demonstrate the cleaning effect of hydrogenation towards hydrocarbon contaminations adsorbed on graphene surfaces.

\subsection{Mechanistic study of the cleaning effect}

\subsubsection{In situ TPD-IR spectroscopy}

The p-doped H-G was previously reported to show n-doping behavior after heating the sample in vacuum to reduce water adsorption at the surface [23]. Taking the observed cleaning effect in H-G, we hypothesize that the adsorption of water on $\mathrm{H}-\mathrm{G}$ surface is closely related to the cleaning effect mentioned above. Therefore, in situ temperature-programmed desorption-infrared spectroscopy (TPD-IR) and near-ambient pressure X-ray photoelectron spectroscopy (NAP-XPS) were further used to mechanistically understand the cleaning effect introduced by the hydrogenation of graphene.

For in situ TPD-IR, a piece of CVD graphene is transferred via a biphasic polymer-free transfer method [25] on one side of an infrared-transparent $\mathrm{BaF}_{2}$ window that is mounted on the tip of a $15 \mathrm{~K}$ cryostat, positioned at the center of a high vacuum (HV) chamber in which the measurements are performed [33]. The HV chamber has a base pressure of $5 \times 10^{-9}$ mbar when the cryostat is on, and residual gas in the chamber is dominated by $\mathrm{H}_{2} \mathrm{O}$ and $\mathrm{H}_{2}$. Sample temperatures are set with $\sim 2 \mathrm{~K}$ precision using thermocouples and a temperature controller. It should be noted that we intentionally set the temperature during water deposition through a separate gas manifold to be higher than the typical thermal desorption temperature of water ice $(\sim 160 \mathrm{~K})$, ensuring that water ice 
does not build up on the sample. Prior to the TPD-IR experiment, the monolayer quality and the lattice integrity of the transferred untreated $\mathrm{G}$ and $\mathrm{H}-\mathrm{G}$ have been confirmed by the sharp and single-Lorentz fitted $\mathrm{G}$ peak $\left(\sim 1,588 \mathrm{~cm}^{-1}\right)$ and 2D peak $\left(\sim 2,670 \mathrm{~cm}^{-1}\right)$ of the Raman spectra in the inset of Figure 2(a,b). Specifically, the D peak $\left(\sim 1,350 \mathrm{~cm}^{-1}\right)$ and D' peak $\left(\sim 1,620 \mathrm{~cm}^{-1}\right)$ as indicators of the presence of defects in graphene lattices are confirmed to be more intensive for $\mathrm{H}-\mathrm{G}$ after $60 \mathrm{~s}$ of hydrogenation [34].

After the cryostat has cooled down, the sample is heated to $324 \mathrm{~K}$ (highest accessible temperature) to degas any water potentially adsorbed to the sample during transfering through the atmosphere. Then the sample is cooled down to $180 \mathrm{~K}$; and at this stage any water adsorption is expected to happen through accretion from the background gas. During water deposition, we observe almost no change in the IR spectra, indicating that during cooling down from $324 \mathrm{~K}$ to $180 \mathrm{~K}$, the graphene surface is already saturated by water. Further exposure to water does not lead to a further increase of $\mathrm{H}_{2} \mathrm{O}$ related bands in the IR spectra. After water deposition, the sample is heated up at a linear ramp rate of $3 \mathrm{~K} / \mathrm{min}$ to $324 \mathrm{~K}$ to desorb the water from the graphene. Typically, physisorbed water desorbs below $180 \mathrm{~K}$, and chemisorbed water desorbs above this temperature [35]. Therefore, IR spectra collected during the heating process records mainly the relative changes induced by the desorption of chemisorbed water molecules at each temperature. A schematic of the HV chamber is shown in Figure 2(d).

Figure 2(a, b) shows the IR absorption spectra of the untreated $\mathrm{G}$ and $\mathrm{H}-\mathrm{G}$ during heating up from $180 \mathrm{~K}$ to $324 \mathrm{~K}$. Each spectrum is calculated with respect to the background scan performed at $180 \mathrm{~K}$ to obtain the desorption signals (see background spectrum in Figure S4(a, b). The two peaks appearing at $\sim 2,924$ and $\sim 2,852 \mathrm{~cm}^{-1}$, respectively, corresponds to the asymmetric and symmetric stretching modes of the $\mathrm{C}-\mathrm{H}$ bond, which has been confirmed to be induced by the adsorbed hydrocarbon contaminations on the graphene surface [36]. Particularly, the broad band appearing at $\sim 3,250 \mathrm{~cm}^{-1}$ is the $\mathrm{OH}$ stretching vibrational modes of water and illustrates the desorption level of chemisorbed water from the graphene surfaces. Increasing the temperature, more water molecules desorb from the surface of $\mathrm{H}-\mathrm{G}$ and result in a larger change in the peak intensities in the IR spectra than that of untreated contaminated G. Figure 2(c) plots the water desorption rates of untreated $\mathrm{G}$ and $\mathrm{H}-\mathrm{G}$ during the heating process from 180 to $320 \mathrm{~K}$. Within the same temperature interval, the amount of water desorbed from $\mathrm{H}-\mathrm{G}$ is almost as two times as that from the untreated one. In other words, such a contrast supports the proposition that hydrogenation treatment increases the water adsorption on graphene surface, assuming that the adsorption energies are similar. Meanwhile, the increasing intensity of $\mathrm{C}-\mathrm{H}$
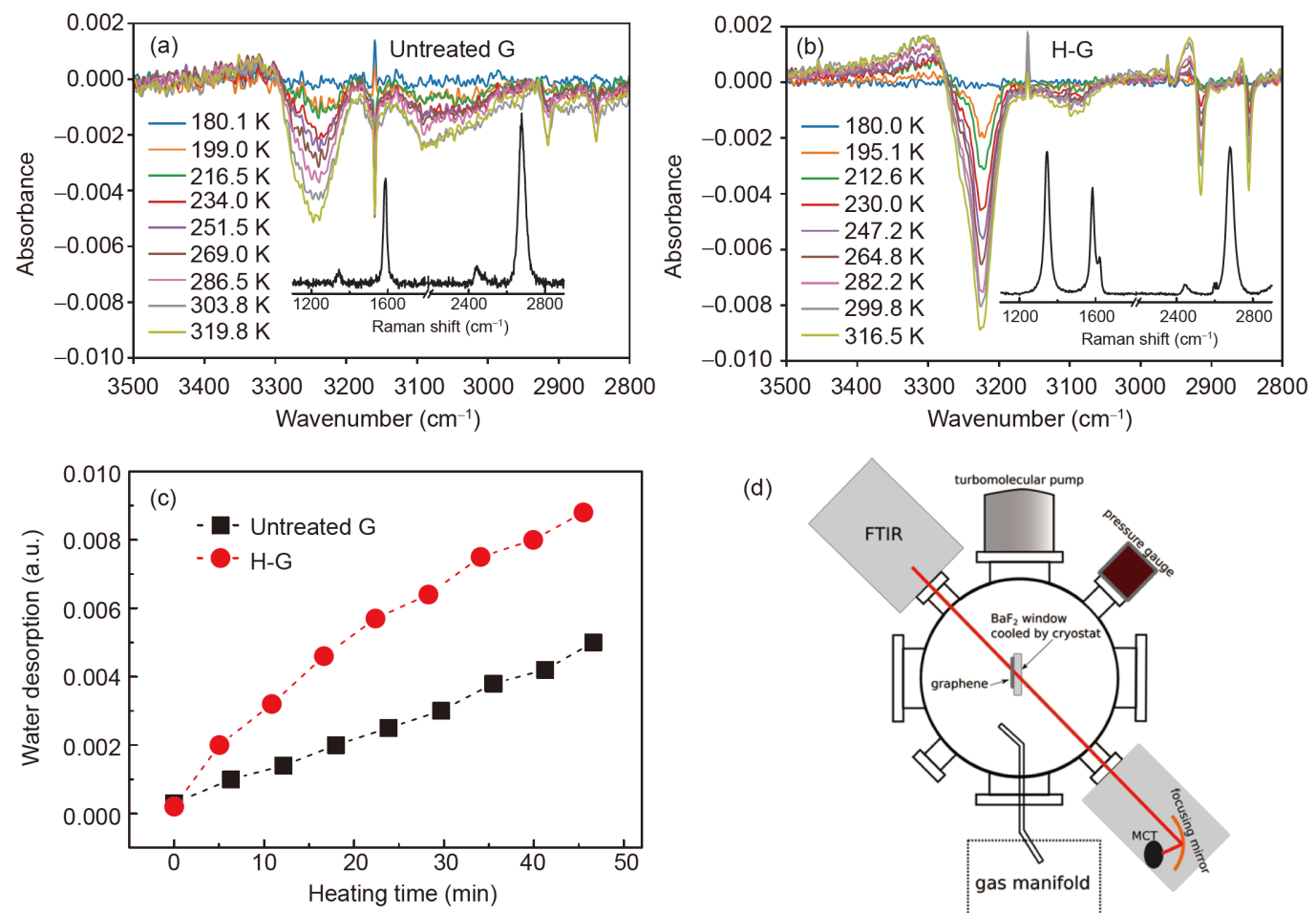

(d)

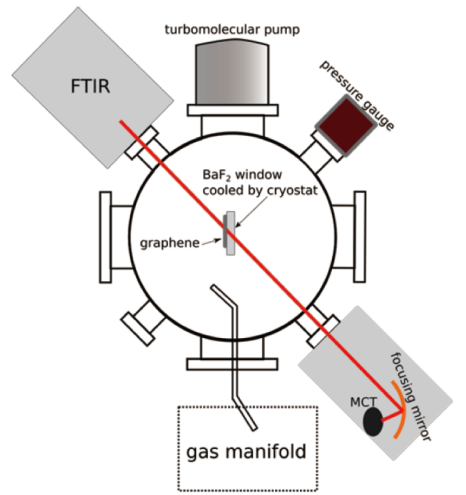

Figure 2 Water adsorption experiments on graphene surfaces characterized by in situ TPD-IR. IR spectra of untreated G (a) and H-G (60 s, b) for temperatures increasing from 180 to $320 \mathrm{~K}$ with increments of 15 to $20 \mathrm{~K}$. The insets in (a, b) are the corresponding Raman spectra of untreated $\mathrm{G}$ and $\mathrm{H}-\mathrm{G}$ on $\mathrm{a} \mathrm{BaF}_{2}$ substrate, respectively. (c) Water desorption rates for untreated $\mathrm{G}$ and $\mathrm{H}-\mathrm{G}$ when temperature increases from 180 to $320 \mathrm{~K}$. The dash lines are guidance for eyes. (d) Schematic of the HV chamber for TPD-IR measurement. All graphene samples are deposited on a BaF 2 window via a polymer-free transfer method. All the graphene samples were characterized within 3 days after preparation (color online). 
stretching peaks in Figure 2(a, b) may originate from the desorption of hydrocarbons adsorbed on the graphene surface. With similar ratio of the peak intensity between $\mathrm{C}-\mathrm{H}$ bonds and the water peak for both untreated $\mathrm{G}$ and $\mathrm{H}-\mathrm{G}(\sim 0.3$ to 0.4 ), we hypothesize that water tends to desorb together with hydrocarbons. The relative stronger $\mathrm{C}-\mathrm{H}$ peak intensities of $\mathrm{H}-\mathrm{G}$ than that of untreated $\mathrm{G}$ can be related to the weakened binding between hydrocarbons and $\mathrm{H}-\mathrm{G}$ due to the presence of more water molecules adsorbed on the graphene lattice. In addition, the IR spectra on H-G when cooling down from room temperature to $180 \mathrm{~K}$ exhibit a similar change in the peak intensity (Figure S4(c)), indicating the reversible water adsorption process.

\subsubsection{Near-ambient pressure X-ray photoelectron spec- troscopy}

In situ NAP-XPS was used to further analyze the affinity of water towards the surface of graphene upon hydrogenation at a water vapor pressure of 1 mbar. The characterization was conducted directly on the CVD graphene as-grown on copper foil to avoid unnecessary transfer-related contaminations. Figure 3(a, b) shows the $\mathrm{C} 1 s$ and $\mathrm{O} 1 s$ spectra for graphene samples under ultra-high vacuum (UHV) at a chamber pressure of $<10^{-9}$ mbar. The $\mathrm{C} 1 \mathrm{~s}$ peak can be deconvoluted into $s p^{2} \mathrm{C}(284.2 \mathrm{eV}), s p^{3} \mathrm{C}(284.8 \mathrm{eV}), \mathrm{C}-\mathrm{O}$ $(286.3 \mathrm{eV})$ and $\mathrm{C}=\mathrm{O}(288.3 \mathrm{eV})$ components. The origin of $s p^{3} \mathrm{C}$ in untreated $\mathrm{G}$ is speculated to be mainly the hydrocarbons adsorbed on the surface. The O $1 s$ spectra in UHV can be fitted by three main components: $\mathrm{Cu}_{2} \mathrm{O}(530.3 \mathrm{eV})$, $\mathrm{C}=\mathrm{O}(531.3 \mathrm{eV})$ and $\mathrm{C}-\mathrm{O}(532.3 \mathrm{eV})$ (Figure 3(b)). The presence of $\mathrm{Cu}_{2} \mathrm{O}$ originates from the underlying copper substrate for graphene growth as the employed Al $K \alpha$ radiation leads to a probing depth of $2-5 \mathrm{~nm}$. At near-ambient pressure conditions in the presence of 1.0 mbar of $\mathrm{H}_{2} \mathrm{O}$, another peak arises at $\sim 535.3 \mathrm{eV}$ (Figure 3(c)), which is ascribed to water molecules chemisorbed at graphene surfaces [37]. The atomic ratios (at.\%) of the $\mathrm{C} 1 s$ components (UHV) in Figure 3(d) show that the ratio of $s p^{2} \mathrm{C}$ keeps increasing when untreated $\mathrm{G}(0 \mathrm{~s} \mathrm{H}-\mathrm{G})$ is hydrogenated (i.e., $30 \mathrm{~s}$ and $60 \mathrm{~s} \mathrm{H}-\mathrm{G})$ and decreases for longer hydrogenation times (i.e., $120 \mathrm{~s} \mathrm{H}-\mathrm{G}$ ). Meanwhile, $s p^{3} \mathrm{C}$ ratios increase monotonously corresponding to the increase of hydrogenation time as shown by Raman spectroscopy (inset of Figure 2 (b)). By contrast, the content of $\mathrm{C}=\mathrm{O}$ and $\mathrm{C}-\mathrm{O}$ both decreases upon hydrogenation. Considering the synchronized ratio variations between $s p^{2} \mathrm{C}$ and $\mathrm{C}-\mathrm{O} / \mathrm{C}=\mathrm{O}$ groups, the rise of $s p^{2} \mathrm{C}$ content (0-60 s H-G) can be mainly ascribed to the chemical reduction and/or removal of oxygenated groups adsorbed at the graphene surface. When continuous hybridization change takes over the reduction/removal effect, the $s p^{2} \mathrm{C}$ ratio starts to drop upon $120 \mathrm{~s}$ hydrogenation. In short, the lattice of hydrogenated graphene, particularly after $30 \mathrm{~s}$ and $60 \mathrm{~s}$ of hydrogenation, contains less oxygenated adsorbates and more $s p^{2} \mathrm{C}$ content than that of the untreated graphene.

To compare the difference of water adsorption for untreated $\mathrm{G}$ and $\mathrm{H}-\mathrm{G}$, we calculated the ratios of the component areas of $\mathrm{H}_{2} \mathrm{O}$ versus $\mathrm{Cu}$ and versus the peak area of $\mathrm{C}$ spectra (Figure 3(e)). The ratios of $\mathrm{H}_{2} \mathrm{O} / \mathrm{Cu}$ are considered to give more reliable details for water adsorption preference than $\mathrm{H}_{2} \mathrm{O} / \mathrm{C}$ ratios because graphene prevents copper to be oxidized. The ratios of $\mathrm{H}_{2} \mathrm{O} / \mathrm{Cu}$ are generally higher in $\mathrm{H}-\mathrm{G}$ $(>15 \%)$ than that of the untreated $\mathrm{G}(14.7 \%)$ with $30 \mathrm{~s} \mathrm{H}-\mathrm{G}$ plateaued $(26.7 \%)$, which suggests that $\mathrm{H}-\mathrm{G}$ presents a higher water affinity. For complement, the $\mathrm{H}_{2} \mathrm{O} / \mathrm{C}$ ratios are higher after $30 \mathrm{~s}$ of hydrogenation (from $17.1 \%$ to $24.7 \%$ ) but drop again upon $60 \mathrm{~s}(16.4 \%)$ to $120 \mathrm{~s}(14.7 \%)$ of hydrogenation. In brief, the ratios of $\mathrm{H}_{2} \mathrm{O} / \mathrm{Cu}$ reach the plateau for medium hydrogen coverage ( $30 \mathrm{~s}$ ) compared to higher coverages (60 and $120 \mathrm{~s}$ ). On one hand, water adsorption on graphene may be saturated at low hydrogenation degree and then tend to decrease with the higher hydrogenation content. On the other hand, other factors impacting the interaction between water and graphene including work function [38], adsorption energy [39], doping effect [40,41], substrate effect [40], to name a few, may also contribute to the observed phenomenon. More detailed insight into the interaction between hydrogenated graphene and water is worth of further investigations. To sum up, hydrogenated graphene presents higher ratios of water adsorption than untreated graphene. Figure 3(f) plots the positive correlation between the surface cleanness and relative ratios of water adsorption for graphene samples, further suggesting that the cleaned surface for H-G exhibits a higher water affinity. Despite of more water adsorption on the graphene surface after hydrogenation (Figure 3 ), the hydrophilicity of hydrogenated graphene measured by water contact angle measurements is similar [42]. Interestingly, a recent theoretical work reports that hydrogenated graphene is more hydrophobic than untreated graphene because of a decrease of surface energy upon hydrogenation [43]. Separately, several experimental work supports that hydrogenation changes graphene from hydrophobic to highly hydrophilic $[2,44]$. These controversial results, indeed, point out the necessity to systematically control and investigate the surface/interface chemistry of graphene, where cleanness plays an important role in understanding the wetting properties of graphene-on-surfaces [41].

The underlying mechanism of the observed cleaning effect on H-G is ascribed to water adsorption. It is well-known that hydrocarbon contaminants interact with graphene via van der Waals interactions to minimize the intrinsically high surface energy of graphene $[8,45]$. As H-G adsorbs more water molecules than untreated graphene, the van der Waals interaction distance between hydrocarbons and graphene is extended, thus leading to less hydrocarbon adsorption or slowing down this process for H-G [45]. Additionally, the 

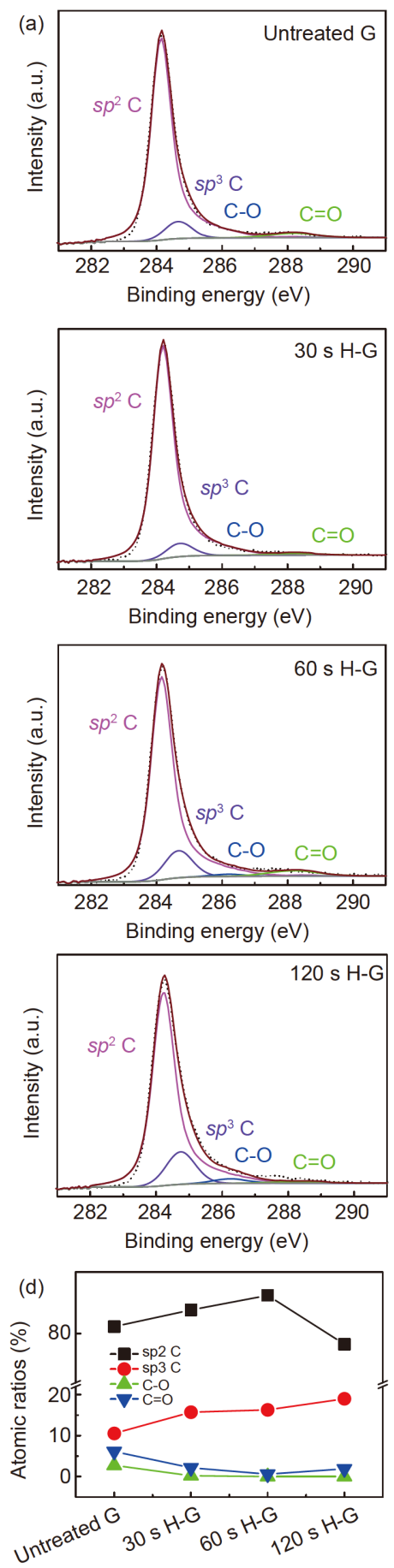
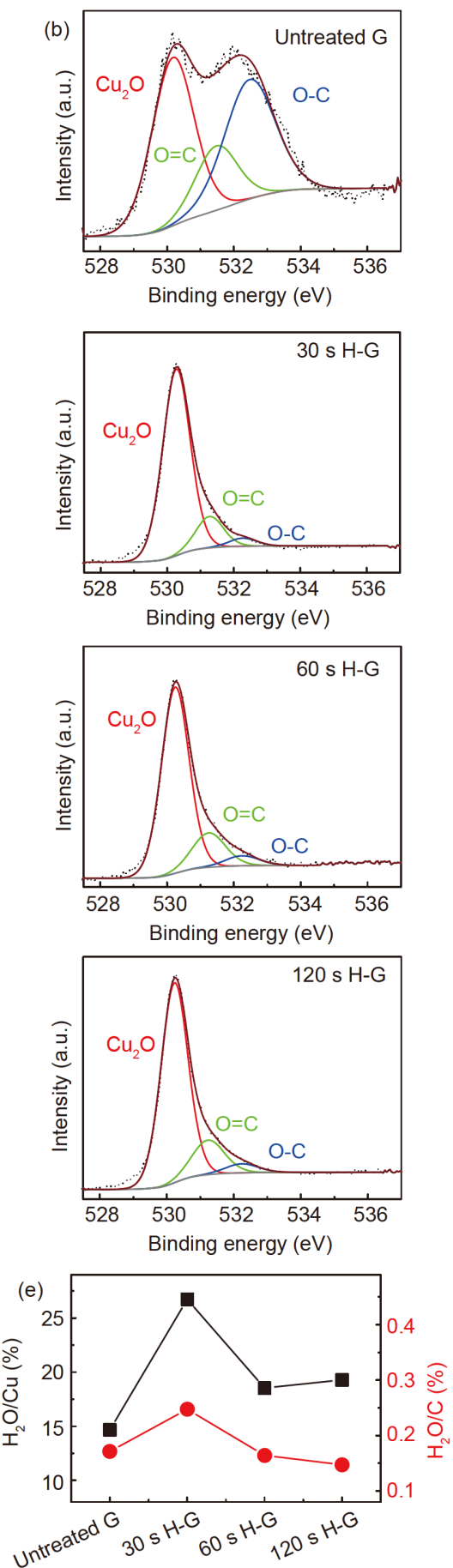
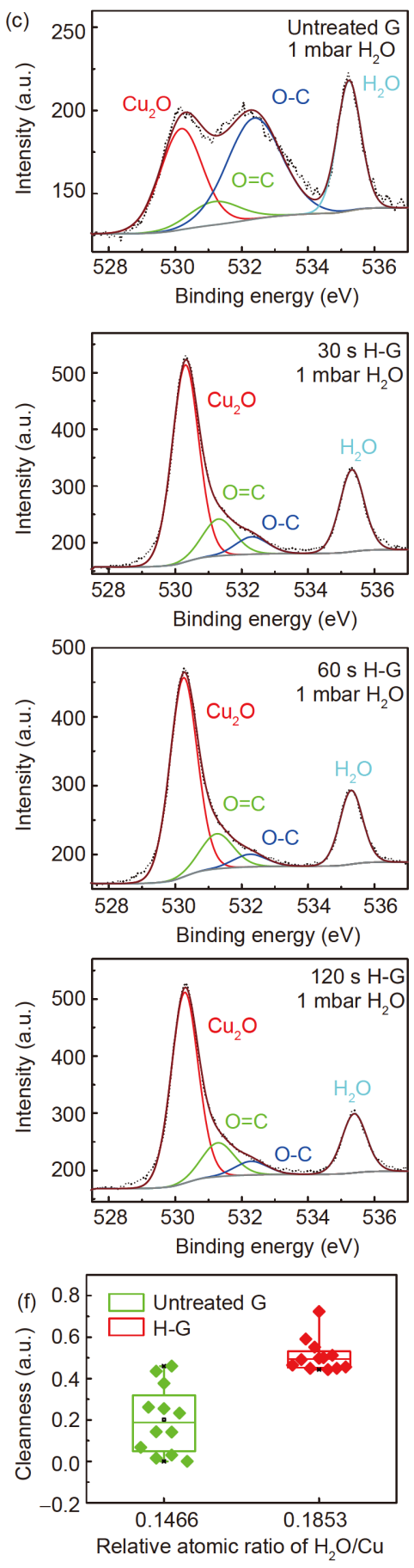

Figure 3 Ultra-high vacuum (UHV) and in situ near-ambient pressure (1 mbar $\mathrm{H}_{2} \mathrm{O}$, NAP-) XPS analysis of untreated and hydrogenated CVD graphene (untreated G and $30 \mathrm{~s}, 60 \mathrm{~s}, 120 \mathrm{~s} \mathrm{H}-\mathrm{G})$. (a) High-resolution carbon (C) $1 \mathrm{~s}$ spectra in UHV. (b) High-resolution oxygen (O) $1 \mathrm{~s}$ spectra in UHV. (c) Highresolution $\mathrm{O} 1 s$ spectra in the presence of 1 mbar of $\mathrm{H}_{2} \mathrm{O}$. (d) Atomic ratios of $\mathrm{C} 1 s$ in $\mathrm{UHV}$. (e) Corresponding atomic ratios of chemisorbed $\mathrm{H}_{2} \mathrm{O}$ versus $\mathrm{Cu}$ (black) and $\mathrm{C}$ (red) obtained from calculating the peak area. (f) Correlation between surface cleanness and water adsorption for untreated $\mathrm{G}$ and $60 \mathrm{~s}$ H-G (color online).

possibility for hydrocarbons to re-contaminate graphene is lowered when water molecules occupy most of the adsorption sites. Therefore, the water adsorption on the clean lattice of $\mathrm{H}-\mathrm{G}$, which is surrounded by hydrocarbons, prevents or at least effectively slows down the process of hydrocarbon contaminations.

\subsection{Dehydrogenated graphene for high quality elec- tronics}

It is known that $\mathrm{H}-\mathrm{G}$ can be reversibly recovered to graphene by annealing under argon atmosphere without structural damage or broken symmetry in the $s p^{2}$ carbon network [21]. 
Therefore, the recovered graphene after dehydrogenation is expected to present a clean and high quality $s p^{2}$ hybridized carbon lattice with few carrier scatterings from defects or charged contaminations than that of untreated graphene [10]. Therefore, CVD graphene on copper foil was first hydrogenated for $60 \mathrm{~s}$ prior to annealing at $350{ }^{\circ} \mathrm{C}$ for $1 \mathrm{~h}$ under an argon atmosphere, referred to as DH-G. Then both untreated G, $60 \mathrm{~s} \mathrm{H}-\mathrm{G}$ and $\mathrm{DH}-\mathrm{G}$ samples were transferred using PMMA on $\mathrm{SiO}_{2} / \mathrm{Si}$ wafers.

Figure 4(a) shows the optical microscopy images of untreated $\mathrm{G}$ and $\mathrm{DH}-\mathrm{G}$ on a $\mathrm{SiO}_{2} / \mathrm{Si}$ wafer. DH-G shows a clean surface with less polymer residues than that of untreated $\mathrm{G}$. The reduced polymer contaminations of transferred DH-G is in line with the reports claiming that CVD graphene on copper with less hydrocarbon contaminations tends to leave less transfer-related polymer residues $[14,16]$. Furthermore, Raman spectroscopy further confirmed the lattice integrity of DH-G (Figure 4(b)). After annealing, DH-G is restored to pristine-like status by recovering the $s p^{3} \mathrm{C}-\mathrm{H}$ bonds to $s p^{2}$ $\mathrm{C}-\mathrm{C}$ bonds, reflected by the dramatically decreased $\mathrm{D}$ peak in comparison with that of H-G. Similar Raman spectra for DH-G and untreated $G$ also corroborate the restored carbon lattices. In particular, the slight change in the width and intensity of the D peak in DH-G can be attributed to the strain in graphene introduced during the annealing process [46]. Strain fields in graphene induced by the underlying support are able to activate intrinsic defects like interstitials and vacancies along the grain boundaries, which normally does not contribute to the D peak in non-strained graphene [47].

Electrical characterizations of untreated $\mathrm{G}$ and DH-G were performed with field-effect transistors architectures to verify if upon dehydrogenation, the charge carrier mobilities in graphene can be restored to the values higher than that of untreated and contaminated graphene. The transport properties of as-prepared GFETs were measured in a liquid gating configuration $(0.1 \mathrm{M} \mathrm{KCl}$ solution containing $10 \mathrm{mM} \mathrm{pH} 8$ Tris as the buffer) at room temperature (Figure 4(c)). Figure 4(d) plots the typical conductance $(G)$ of a untreated G, H-G $(60 \mathrm{~s})$ and a DH-G as a function of the gate voltage $\left(V_{\mathrm{g}}\right)$. As $\mathrm{H}-s p^{3}$ defects introduce short-range scattering into the lattice [12], H-G shows degraded conductivity and mobilities $\left(\sim 160-180 \mathrm{~cm}^{2} \mathrm{~V}^{-1} \mathrm{~s}^{-1}\right)$ compared to untreated G $\left(\sim 500-840 \mathrm{~cm}^{2} \mathrm{~V}^{-1} \mathrm{~s}^{-1}\right)$. In contrast to untreated G, DH-G exhibits a higher minimum conductance $\left(G_{\min }\right)$ value at the charge neutrality point (CNP) and p-doping behavior. On one hand, such a contrast can be attributed to the reduction of scattering events induced by hydrocarbons in DH-G [48], as $G_{\min }$ is dominated by the density of charged impurities according to the Boltzmann theory $[49,50]$. On the other hand, the observed contrast in $G_{\min }$ and p-doping effect are also related to the residue water molecules on DH-G as the physisorption of water is reported to slightly increase the minimum conductance while induce p-doping effect in grapheme [23]. Considering short duration in the annealing step $(\sim 1 \mathrm{~h})$ to dehydrogenate $\mathrm{H}-\mathrm{G}$, the pre-existing water molecules on $\mathrm{H}-\mathrm{G}$ are likely to retain on the surface of DH-G after annealing. From the $G\left(V_{\mathrm{g}}\right)$ curves of six different GFETs, the mobilities for DH-G are (high) standard for CVD graphene, i.e., from $\sim 1,150$ to $\sim 1,630 \mathrm{~cm}^{2} \mathrm{~V}^{-1} \mathrm{~s}^{-1}$ (Figure 4(e)), which are about two to three-fold higher than that of the untreated contaminated $\mathrm{G}$ we started with $\left(\sim 500-840 \mathrm{~cm}^{2} \mathrm{~V}^{-1} \mathrm{~s}^{-1}\right)$.

Given that the adsorbed water molecules decrease the
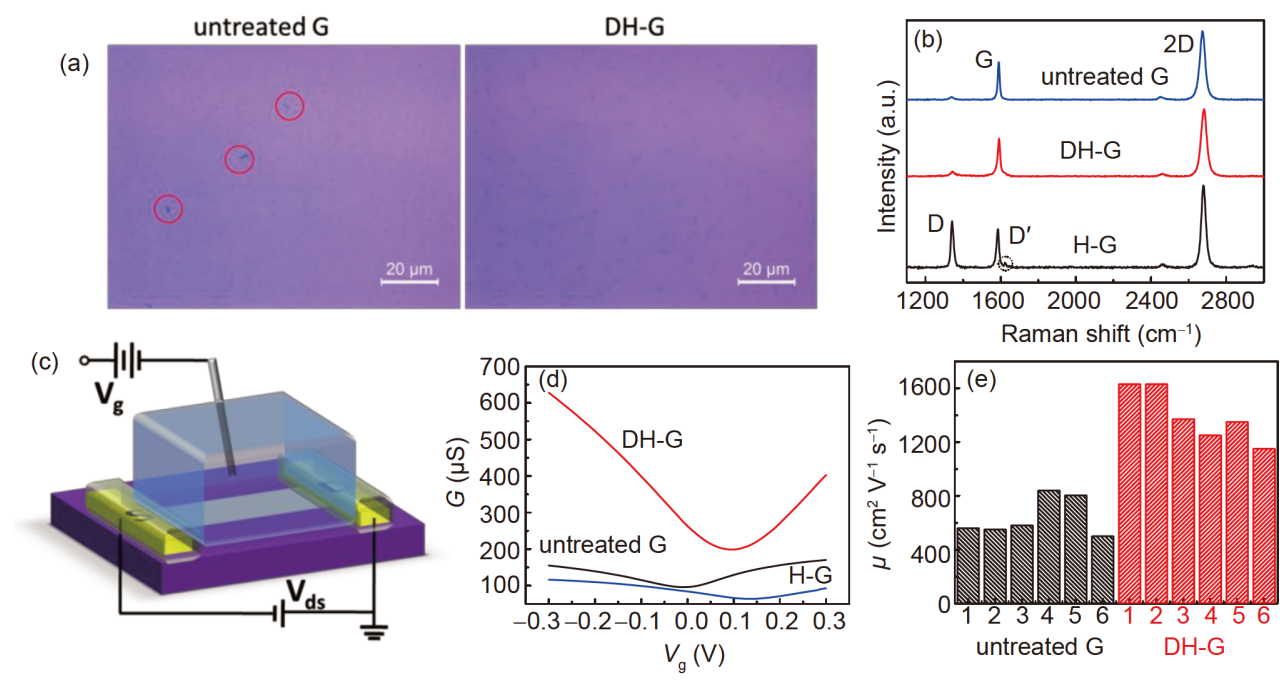

Figure 4 Optical and electrical properties of graphene after dehydrogenation. (a) Optical microscopy image of untreated G and DH-G transferred on a Si wafer. Red circles indicate spots contaminated with polymer residues. (b) Raman spectra of $60 \mathrm{~s} \mathrm{H}-\mathrm{G}, \mathrm{DH}-\mathrm{G}$ after annealing at $350{ }^{\circ} \mathrm{C}$ for $1 \mathrm{~h}$, and untreated $\mathrm{G}$ on a $\mathrm{Si}$ wafer. The spectra were recorded using a $2.33 \mathrm{eV}(532 \mathrm{~nm}$ ) laser excitation. (c) Schematic of the GFET fabricated in a liquid gating configuration. (d) Conductance $(G)$ as a function of gate voltage $\left(V_{\mathrm{g}}\right)$ for untreated G, H-G $(60 \mathrm{~s})$ and DH-G transferred on a Si wafer. (e) Comparison of mobility $(\mu)$ values for untreated G and DH-G. All the measurements were done at room temperature in ambient conditions. All the graphene samples for GFETs were characterized within 3 days after preparation (color online). 
mobility of graphene by increasing its carrier concentration [51], the improved mobilities in DH-G may originate from the less scattering from the adsorbed hydrocarbons. XPS characterization was performed to further confirm the high crystallinity and surface cleanness of DH-G (Figure S5). The atomic ratio of $s p^{2} \mathrm{C}$ in the $\mathrm{C} 1 s$ peak is around $96 \%$ for $\mathrm{DH}$ $\mathrm{G}$, which is higher than that of untreated $\mathrm{G}(89.2 \%)$. It is found that the content of hydrocarbon contaminations (mainly represented by $s p^{3} \mathrm{C}$ ) is significantly reduced from $2.7 \%$ for G to $0 \%$ for DH-G (Figure S5(c)). The content of other carbon-oxygen groups in graphene also decreased after the reversible hydrogenation-dehydrogenation (i.e., reduction of oxidized groups by hydrogen radicals). Therefore, reversible dehydrogenation can effectively yield clean graphene since hydrogenation first removes hydrocarbon contaminations while chemically functionalizing graphene, and the following dehydrogenation annealing step maintains the surface cleanness when recovering the lattice from $s p^{3}$ to $s p^{2}$ hybridization. More importantly, the hydrogenation-dehydrogenation strategy could yield superior graphene for electronic devices exhibiting good mobility and surface purity. For the practical operation, delicate design and setup, i.e., transferring the processed graphene in a clean protection atmosphere, minimizing the exposure to air or containers made by hydrocarbons, slowing down the re-deposition of hydrocarbons in low temperature, could also be taken into account for highly sensitive device fabrication.

\section{Conclusions}

Graphene as a two-dimensional surface is prone to be contaminated by airborne hydrocarbons to minimize its surface energy. Confirmed by HRTEM images and XPS characterizations, hydrogenation of graphene can remove partially the adsorbed hydrocarbons without damaging the lattice integrity and crystallinity. Quantitative analysis of TEM images shows that hydrogenated graphene generally presents and keeps a cleaner surface than the untreated, contaminated samples. Moreover, a water desorption experiment using in situ TPD-IR and NAP-XPS characterizations in the presence of 1 mbar $\mathrm{H}_{2} \mathrm{O}$ further confirmed that graphene adsorbs more water after it has experienced a hydrogenation treatment. The mechanistic investigation shows that hydrogen radicals first remove surface-adsorbed hydrocarbons and then chemically functionalize the underlying graphene lattice from $s p^{2}$ to $s p^{3}$, resulting in an increased water adsorption of $\mathrm{H}-\mathrm{G}$ to prevent further hydrocarbon contaminations. The reversible dehydrogenation of $\mathrm{H}-\mathrm{G}$ recovers the intrinsic lattice, yielding a cleaner graphene with higher carrier mobilities than that in the untreated G. We therefore believe that reversible (de)hydrogenation treatment of CVD graphene can be employed to fabricate high-performance graphene electronic devices with sustainable surface cleanness. Moreover, the low level of contaminations from hydrocarbons allows a better control of the surface chemistry of graphene, facilitating more surface-/interface-related studies and applications including wettability and sensors.

Acknowledgements This work was supported by the Chinese Scholarship Council (201406890016), NWA route 'meten \& detecteren', the European Research Council under the European Union's Seventh Framework Program (FP/2007-2013)/ERC Grant Agreement No. 335879 project acronym 'Biographene', the Netherlands Organization for Scientific Research (Vidi 723.013.007). L. Wu and J. P. Hofmann acknowledge funding from The Netherlands Organization for Scientific Research (NWO) and cofinancing by Shell Global Solutions International B.V. for the project 13CO2-6. E.J.M. H. and V.M. acknowledge support by the Netherlands Center for Multiscale Catalytic Energy Conversion (MCEC), an NWO Gravitation program funded by the Ministry of Education, Culture and Science of the government of the Netherlands and a Vici grant of the NWO.

Conflict of interest The authors declare no conflict of interest.

Supporting information The supporting information is available online at http://chem.scichina.com and http://link.springer.com/journal/11426. The supporting materials are published as submitted, without typesetting or editing. The responsibility for scientific accuracy and content remains entirely with the authors.

Open Access This article is licensed under a Creative Commons Attribution 4.0 International License, which permits use, sharing, adaptation, distribution and reproduction in any medium or format, as long as you give appropriate credit to the original author(s) and the source, provide a link to the Creative Commons licence, and indicate if changes were made. The images or other third party material in this article are included in the article's Creative Commons licence, unless indicated otherwise in a credit line to the material. If material is not included in the article's Creative Commons licence and your intended use is not permitted by statutory regulation or exceeds the permitted use, you will need to obtain permission directly from the copyright holder. To view a copy of this licence, visit http://creativecommons.org/licenses/by/4.0/.

1 Surwade SP, Smirnov SN, Vlassiouk IV, Unocic RR, Veith GM, Dai S, Mahurin SM. Nat Nanotech, 2015, 10: 459-464

2 Russo CJ, Passmore LA. Nat Methods, 2014, 11: 649-652

3 Kim Y, Cruz SS, Lee K, Alawode BO, Choi C, Song Y, Johnson JM, Heidelberger C, Kong W, Choi S, Qiao K, Almansouri I, Fitzgerald EA, Kong J, Kolpak AM, Hwang J, Kim J. Nature, 2017, 544: 340343

4 Zhang Z, Du J, Zhang D, Sun H, Yin L, Ma L, Chen J, Ma D, Cheng HM, Ren W. Nat Commun, 2017, 8: 14560

5 Lin L, Zhang J, Su H, Li J, Sun L, Wang Z, Xu F, Liu C, Lopatin S, Zhu Y, Jia K, Chen S, Rui D, Sun J, Xue R, Gao P, Kang N, Han Y, Xu HQ, Cao Y, Novoselov KS, Tian Z, Ren B, Peng H, Liu Z. Nat Commun, 2019, 10: 1912

6 Jia K, Zhang J, Lin L, Li Z, Gao J, Sun L, Xue R, Li J, Kang N, Luo Z, Rummeli MH, Peng H, Liu Z. J Am Chem Soc, 2019, 141: 7670-7674

7 Pettes MT, Jo I, Yao Z, Shi L. Nano Lett, 2011, 11: 1195-1200

8 Li Z, Wang Y, Kozbial A, Shenoy G, Zhou F, McGinley R, Ireland P, Morganstein B, Kunkel A, Surwade SP, Li L, Liu H. Nat Mater, 2013, 12: 925-931

9 Novoselov KS, Fal'ko VI, Colombo L, Gellert PR, Schwab MG, Kim K. Nature, 2012, 490: 192-200

10 Chen JH, Jang C, Xiao S, Ishigami M, Fuhrer MS. Nat Nanotech, 
2008, 3: 206-209

11 Patel AN, Collignon MG, O'Connell MA, Hung WOY, McKelvey K, Macpherson JV, Unwin PR. J Am Chem Soc, 2012, 134: 20117-20130

12 Jiang L, Fu W, Birdja YY, Koper MTM, Schneider GF. Nat Commun, 2018, 9: 793

13 Moser J, Barreiro A, Bachtold A. Appl Phys Lett, 2007, 91: 163513

14 Sun L, Lin L, Wang Z, Rui D, Yu Z, Zhang J, Li Y, Liu X, Jia K, Wang K, Zheng L, Deng B, Ma T, Kang N, Xu H, Novoselov KS, Peng H, Liu Z. Adv Mater, 2019, 31: 1902978

15 Choi WJ, Chung YJ, Park S, Yang CS, Lee YK, An KS, Lee YS, Lee JO. Adv Mater, 2014, 26: 637-644

16 Zhang J, Jia K, Lin L, Zhao W, Quang HT, Sun L, Li T, Li Z, Liu X, Zheng L, Xue R, Gao J, Luo Z, Rummeli MH, Yuan Q, Peng H, Liu Z. Angew Chem Int Ed, 2019, 58: 14446-14451

17 Ferrah D, Renault O, Petit-Etienne C, Okuno H, Berne C, Bouchiat V, Cunge G. Surf Interface Anal, 2016, 48: 451-455

18 Cunge G, Ferrah D, Petit-Etienne C, Davydova A, Okuno H, Kalita D, Bouchiat V, Renault O. J Appl Phys, 2015, 118: 123302

19 Harpale A, Panesi M, Chew HB. Phys Rev B, 2016, 93: 035416

20 Despiau-Pujo E, Davydova A, Cunge G, Graves DB. Plasma Chem Plasma Process, 2016, 36: 213-229

21 Son J, Lee S, Kim SJ, Park BC, Lee HK, Kim S, Kim JH, Hong BH, Hong J. Nat Commun, 2016, 7: 13261

22 Jiang L. Dissertation for the Doctoral Degree. Leiden: Leiden University, 2020

23 Matis BR, Burgess JS, Bulat FA, Friedman AL, Houston BH, Baldwin JW. ACS Nano, 2012, 6: 17-22

24 Wu S, Yang R, Shi D, Zhang G. Nanoscale, 2012, 4: 2005-2009

25 Belyaeva LA, Fu W, Arjmandi-Tash H, Schneider GF. ACS Cent Sci, 2016, 2: 904-909

26 Nguyen L, Tao FF. Rev Sci Instruments, 2016, 87: 064101

27 Huang PY, Ruiz-Vargas CS, van der Zande AM, Whitney WS, Levendorf MP, Kevek JW, Garg S, Alden JS, Hustedt CJ, Zhu Y, Park J, McEuen PL, Muller DA. Nature, 2011, 469: 389-392

28 Algara-Siller G, Lehtinen O, Turchanin A, Kaiser U. Appl Phys Lett, 2014, 104: 153115

29 Xu Q, Wu MY, Schneider GF, Houben L, Malladi SK, Dekker C, Yucelen E, Dunin-Borkowski RE, Zandbergen HW. ACS Nano, 2013, 7: $1566-1572$

30 Felten A, McManus D, Rice C, Nittler L, Pireaux JJ, Casiraghi C. Appl
Phys Lett, 2014, 105: 183104

31 Eckmann A, Felten A, Mishchenko A, Britnell L, Krupke R, Novoselov KS, Casiraghi C. Nano Lett, 2012, 12: 3925-3930

32 Regan W, Alem N, Alemán B, Geng B, Girit Ç, Maserati L, Wang F, Crommie M, Zettl A. Appl Phys Lett, 2010, 96: 113102

33 Kofman V, Witlox MJA, Bouwman J, Ten Kate IL, Linnartz H. Rev Sci Instruments, 2018, 89: 053111

34 Ferrari AC, Basko DM. Nat Nanotech, 2013, 8: 235-246

35 Jo SK, Kiss J, Polanco JA, White JM. Surf Sci, 1991, 253: 233-244

36 Salim M, Hurst J, Montgomery M, Tolman N, Liu H. J Electron Spectr Related Phenomena, 2019, 235: 8-15

37 Ganguly A, Sharma S, Papakonstantinou P, Hamilton J. J Phys Chem C, 2011, 115: 17009-17019

38 Jiang L, Wang J, Liu P, Song W, He B. RSC Adv, 2018, 8: 1121611221

39 Brandenburg JG, Zen A, Fitzner M, Ramberger B, Kresse G, Tsatsoulis T, Grüneis A, Michaelides A, Alfè D. J Phys Chem Lett, 2019, 10: $358-368$

40 Chakradhar A, Sivapragasam N, Nayakasinghe MT, Burghaus U. Chem Commun, 2015, 51: 11463-11466

41 Belyaeva LA, Schneider GF. Surf Sci Rep, 2020, 75: 100482

42 Prydatko AV, Belyaeva LA, Jiang L, Lima LMC, Schneider GF. Nat Commun, 2018, 9: 4185

43 Taqieddin A, Heiranian M, Aluru NR. J Phys Chem C, 2020, 124: 21467-21475

44 Son J, Lee JY, Han N, Cha J, Choi J, Kwon J, Nam SW, Yoo KH, Lee GH, Hong J. Nano Lett, 2020, 20: 5625-5631

45 Li Z, Kozbial A, Nioradze N, Parobek D, Shenoy GJ, Salim M, Amemiya S, Li L, Liu H. ACS Nano, 2015, 10: 349-359

46 Belyaeva LA, Jiang L, Soleimani A, Methorst J, Risselada HJ, Schneider GF. Nat Commun, 2020, 11: 898

47 Wang B, Puzyrev Y, Pantelides ST. Carbon, 2011, 49: 3983-3988

48 Newaz AKM, Puzyrev YS, Wang B, Pantelides ST, Bolotin KI. Nat Commun, 2012, 3: 734

49 Adam S, Hwang EH, Galitski VM, Das Sarma S. Proc Natl Acad Sci USA, 2007, 104: 18392-18397

50 Chen F, Qing Q, Xia J, Li J, Tao N. J Am Chem Soc, 2009, 131: 9908 9909

51 Zhu W, Perebeinos V, Freitag M, Avouris P. Phys Rev B, 2009, 80: 235402 